\title{
Charge localization instability in a highly deformable dielectric elastomer
}

\section{Citation}

Lu, Tongqing, Christoph Keplinger, Nikita Arnold, Siegfried Bauer, and Zhigang Suo. 2014. "Charge Localization Instability in a Highly Deformable Dielectric Elastomer." Appl. Phys. Lett. 104 (2) (January 13): 022905. doi:10.1063/1.4862325.

\section{Published Version}

doi:10.1063/1.4862325

\section{Permanent link}

http://nrs.harvard.edu/urn-3:HUL.InstRepos:13909888

\section{Terms of Use}

This article was downloaded from Harvard University's DASH repository, and is made available under the terms and conditions applicable to Open Access Policy Articles, as set forth at http:// nrs.harvard.edu/urn-3:HUL.InstRepos:dash.current.terms-of-use\#OAP

\section{Share Your Story}

The Harvard community has made this article openly available.

Please share how this access benefits you. Submit a story.

Accessibility 


\title{
Charge localization instability in a highly deformable dielectric elastomer
}

Tongqing Lư ${ }^{1,2}$, Christoph Keplinger ${ }^{2}$, Nikita Arnold33,4, Siegfried Bauer3,a, and Zhigang Suo ${ }^{2, a}$

${ }^{1}$ State Key Lab for Strength and Vibration of Mechanical Structures, International Center for Applied Mechanics, School of Aerospace Engineering, Xi'an Jiaotong University, Xi'an 710049, China

${ }^{2}$ School of Engineering and Applied Sciences, Kavli Institute of Bionano Science and Technology, Harvard University, Cambridge, MA 02138, USA

${ }^{3}$ Soft Matter Physics, 4 Institute of Experimental Physics, Johannes Kepler University, Altenbergerstrasse 69, A-4040 Linz, Austria

\begin{abstract}
This paper shows that a highly deformable capacitor made of a soft dielectric and two conformal electrodes can switch between two states discontinuously, by a first-order transition, as the total charge varies gradually. When the total charge is small, it spreads evenly over the area of the capacitor, and the capacitor deforms homogeneously. When the total charge is large, it localizes in a small region of the capacitor, and this region thins down preferentially. The capacitor will survive the localization without electrical breakdown if the area of the electrode is small. Such a bistable system may lead to useful devices.
\end{abstract}

a Corresponding authors, emails: sbauer@jku.at, suo@seas.harvard.edu 
A fundamental challenge in the emerging field of soft machines is to develop actuators that mimic neuromuscular systems, inducing rapid, powerful, quiet movements in response to electrical stimulation. ${ }^{1-5} \quad$ A promising technology relies on highly deformable capacitors made of dielectric elastomers. ${ }^{6-8}$ Demonstrated uses are wide ranging; in soft robots, ${ }^{9}$ MEMS, ${ }^{10-13}$ tunable lenses, ${ }^{14,15}$ haptic interfaces,${ }^{16}$ and transparent loudspeakers. ${ }^{17}$ Also under development are generators that harvest energy from animal movements, structural vibrations, and ocean waves. $^{18-21}$

The highly deformable capacitors exhibit rich nonlinear behavior, which depends on the method of electrical stimulation. In voltage-controlled actuation, the electric field across the thickness increases as the thickness of the dielectric reduces. This positive feedback may cause pull-in instability and limit actuation strain. ${ }^{22,23}$ By contrast, giant actuation strain is achieved by corona charging. ${ }^{24}$ In this method of actuation, no electrodes cover the dielectric, so that charges on the surfaces of the dielectric are immobile. Such charge-controlled actuation is stable and does not suffer pull-in instability. ${ }^{24,25}$ Corona charging nicely demonstrates a principle, but is of limited use as a method of actuation, because it is slow and energy-inefficient, and cannot be made compact.

Here we consider a more practical type of charge-controlled actuation, where the dielectric elastomer is sandwiched between two conformal electrodes, such as carbon grease. We show that the capacitor can switch between two states when the total charge on the capacitor is varied gradually. The charge spreads uniformly over the area of the capacitor when the total charge is small, but localizes in a small region of the capacitor when the total charge is large. The switch from the homogeneous state to the localized state is discontinuous, corresponding to a first-order transition. The charge-localized capacitor will suffer electrical breakdown if the area of the electrodes is large, but will stabilize if the area of the electrodes is small.

We begin with homogeneous charge-controlled actuation, in which the charge is immobilized on the surfaces of the dielectric, e.g., deposited by corona charging (Fig. 1a). The theory of dielectric elastomers has been reviewed recently. ${ }^{26}$ In the reference state, the 
capacitor is uncharged and undeformed, and has area $A$ and thickness $H$. In the actuated state, the capacitor has voltage $\Phi$, charge $Q$, area $a$ and thickness $h$. The deformation is equal-biaxial in the plane of the capacitor, with the stretch $\lambda=\sqrt{a / A}$. For such a thin-membrane capacitor, the electric field is $E=\Phi / h$, and the electric displacement is $D=Q / a$. The charged capacitor by itself is a closed thermodynamic system. We adopt the model of ideal dielectric elastomer, and assume the Helmholtz free energy density of the form: ${ }^{27}$

$$
W=\frac{\mu}{2}\left(2 \lambda^{2}+\lambda^{-4}-3\right)+\frac{D^{2}}{2 \varepsilon},
$$

where $\mu$ is the shear modulus, and $\varepsilon$ the (absolute) permittivity, both of which are material constants. The free energy is a sum of two parts: elastic energy described by the neo-Hookean model, and electrostatic energy described by the linear dielectric model, $D=\varepsilon E$. Furthermore, the model assumes incompressibility of the elastomer, $H A=h a$, so that $h=H \lambda^{-2}$. The free energy of the charge-controlled capacitor is $F_{Q}=H A W$, namely,

$$
F_{Q}=\frac{\mu H A}{2}\left(2 \lambda^{2}+\lambda^{-4}-3\right)+\frac{Q^{2} H}{2 \varepsilon A} \lambda^{-4} .
$$

At a fixed charge, the free energy as a function of the stretch, $F_{Q}(\lambda)$, has a minimum (Fig. 1a).

Setting $d F_{Q} / d \lambda=0$ with $Q$ held constant, we find the equation of state:

$$
Q^{2}=\mu \varepsilon A^{2}\left(\lambda^{6}-1\right)
$$

This charge-stretch relation is monotonic. For a fixed charge, Eq. (3) determines a unique value of stretch corresponding to a stable state of equilibrium. This analysis reproduces an existing result: homogenous, charge-controlled actuation is stable. ${ }^{25}$

In voltage-controlled actuation, the actuator connects to a charge reservoir at constant voltage (Fig. 1b). The free energy of the capacitor is $H A W$, and the potential energy of the charge reservoir reduces by $\Phi Q$. Consequently, the free energy of the composite system (the capacitor and the charge reservoir) is $F_{\Phi}=H A W-\Phi Q$. Recalling that $\Phi=E h, h=H \lambda^{-2}$, 
$Q=D a, a=\lambda^{2} A$ and $D=\varepsilon E$, we find that $Q=\varepsilon \lambda^{4} A \Phi / H$, where the capacitance $\varepsilon \lambda^{4} A / H$ increases with the stretch. The free energy of the composite system is

$$
F_{\Phi}=\frac{\mu H A}{2}\left(2 \lambda^{2}+\lambda^{-4}-3\right)-\frac{\varepsilon A \Phi^{2}}{2 H} \lambda^{4}
$$

At a fixed voltage, the free energy as a function of the stretch, $F_{\Phi}(\lambda)$, has a minimum and a maximum (Fig. 1b). Setting $d F_{\Phi} / d \lambda=0$ with $\Phi$ held constant, we find the equation of state:

$$
\varepsilon \Phi^{2}=\mu H^{2}\left(\lambda^{-2}-\lambda^{-8}\right)
$$

This voltage-stretch relation is not monotonic, and has a maximum. For a fixed voltage below this maximum, (5) determines two values of stretch, the smaller one corresponding to a stable, and the larger one to an unstable state of equilibrium. This analysis reproduces another existing result: voltage-controlled actuation can undergo pull-in instability. ${ }^{22}$

Pull-in instability in voltage-controlled actuation has been studied for different geometries, such as flat membranes, spherical balloons, tubular balloons, ${ }^{23}, 28-30$ and under different loading conditions, such as equal-biaxial loading, uniaxial loading, and pure-shear conditions..$^{31-33}$ Pull-in instability often leads to electrical breakdown, and should be eliminated in the design of actuators. ${ }^{23}$ On the other hand, one can design actuators to operate near the verge of instability, leading to safe, giant actuation. ${ }^{34}$ Voltage-actuated areal expansions over $1000 \%$ have been demonstrated. ${ }^{29}$

Recalling that $\Phi=E h, h=H \lambda^{-2}, Q=D a, a=\lambda^{2} A$ and $D=\varepsilon E$, one can confirm that the two equations of state, (3) and (5), are identical. We plot the equation of state on the charge-voltage plane using both (3) and (5) by regarding the stretch as a parameter. The charge-voltage curve is not monotonic (Fig. 1c). For charge-controlled actuation, charge is gradually added to the homogeneous system (e.g., by corona charging), a single stable state changes continuously, and no instability occurs. The maximum actuation stretch is limited by electrical breakdown field. For voltage-controlled actuation, as the voltage $\Phi$ ramps up and reaches its maximum value, two branches of solutions merge. No state of equilibrium exists for 
higher voltages, resulting in electromechanical instability. Setting $d \Phi / d \lambda=0$ in (5), we obtain the critical stretch $\lambda_{c}=2^{1 / 3}$, the critical voltage $\Phi_{c}=2^{-4 / 3} H \sqrt{3 \mu / \varepsilon}$, the critical charge $Q_{c}=A \sqrt{3 \varepsilon \mu}$, and the critical electric field $E_{c}=2^{-2 / 3} \sqrt{3 \mu / \varepsilon} \cdot{ }^{22}$ A membrane of neo-Hookean material will become thinner and thinner, leading to electrical breakdown. Consequently, voltage-controlled actuation is limited by the critical state. Incidentally, for more realistic models with strain-stiffening, a second stable high-stretch state appears which often lies beyond the condition of electrical breakdown. ${ }^{23}$

We next turn to localization in charge-controlled actuation (Fig.2a). We seek the condition of instability. The entire membrane is electrically connected. Once a small region of the membrane loses stability and becomes thinner than the rest of the membrane, charge will flow to the small region, and the positive feedback will lead to localization. The rest of the membrane will not thin down. Because the small region is constrained by the rest of the membrane, after charge localization, the small region will have larger stretch in area than the surrounding membrane. Consequently, the small region may form wrinkles. In this paper, we do not analyze the critical condition for the onset of wrinkles, but simply assume that the in-plane stress is zero everywhere in the membrane. For a dielectric sandwiched between two electrodes, even when the total charge on the capacitor is fixed, charge can flow in the electrodes and localize in a small region of the capacitor. In a simplified model we represent the capacitor by two regions, which are electrically connected and have the same voltage $\Phi$ (Fig. 2b). In the reference state, the capacitor is uncharged and undeformed, the two regions have the same thickness $H$, the small region has area $A_{S}$, the large region has area $A_{L}$, and the capacitor has the total area $A=A_{S}+A_{L}$. In the actuated state, each region can undergo its own homogeneous deformation. They have areas $a_{S}$ and $a_{L}$, and the in-plane equal-biaxial stretches are $\lambda_{S}=\sqrt{a_{S} / A_{S}}$ and $\lambda_{L}=\sqrt{a_{L} / A_{L}}$, respectively. This model neglects the effect of the boundary between the two regions. The charges on the two capacitors are $Q_{L}=a_{L} D_{L}=\varepsilon \lambda_{L}^{4} A_{L} \Phi / H$ and 
$Q_{S}=a_{S} D_{S}=\varepsilon \lambda_{S}^{4} A_{S} \Phi / H$. Once the capacitor receives electric charge, the power source is disconnected, so that the total charge $Q$ on the two regions is fixed, and is the sum of the charges on the two regions, $Q=Q_{L}+Q_{S}$, namely,

$$
Q=\varepsilon\left(\lambda_{L}^{4} A_{L}+\lambda_{S}^{4} A_{S}\right) \Phi / H
$$

The two regions are two capacitors in parallel, and the coefficient of the charge-voltage relation (6) is their combined capacitance, $C=\varepsilon\left(\lambda_{L}^{4} A_{L}+\lambda_{S}^{4} A_{S}\right) / H$. The free energies per unit volume of the two regions, $W_{L}$ and $W_{S}$, are calculated from (1) using quantities of the two regions. The free energy of this composite system is a sum of the free energies of both regions, $F=H A_{L} W_{L}+H A_{S} W_{S}$, namely,

$$
F=\frac{\mu H A_{L}}{2}\left(2 \lambda_{L}^{2}+\lambda_{L}^{-4}-3\right)+\frac{\mu H A_{S}}{2}\left(2 \lambda_{S}^{2}+\lambda_{S}^{-4}-3\right)+\frac{H Q^{2}}{2 \varepsilon\left(\lambda_{L}^{4} A_{L}+\lambda_{S}^{4} A_{S}\right)}
$$

The first two terms are due to elasticity of the two regions, while the last term is the total electrostatic energy. The free energy of the two-region system is a function of the two stretches $\left(\lambda_{S}, \lambda_{L}\right)$, and the behavior of this function depends on the value of the total charge $Q$. At a small total charge, the free energy function has only one minimum, corresponding to a homogeneous, stable state of equilibrium (Fig. 3a). At an intermediate total charge, two additional extrema (one minimum and one saddle point) appear, corresponding to two inhomogeneous states of equilibrium (Fig. 3b). At a large total charge, the homogeneous state becomes a saddle point, and the system will stabilize at an inhomogeneous state (Fig. 3c).

Setting $\partial F\left(\lambda_{L}, \lambda_{S}\right) / \partial \lambda_{L}=\partial F\left(\lambda_{L}, \lambda_{S}\right) / \partial \lambda_{S}=0$ in (7) with the total charge $Q$ held constant, we obtain two equations of state:

$$
\begin{aligned}
& \varepsilon \Phi^{2}=\mu H^{2}\left(\lambda_{L}^{-2}-\lambda_{L}^{-8}\right), \\
& \varepsilon \Phi^{2}=\mu H^{2}\left(\lambda_{S}^{-2}-\lambda_{S}^{-8}\right) .
\end{aligned}
$$

At a given total charge $Q,(6),(8)$ and (9) form a set of nonlinear equations for three unknowns: 
$\lambda_{L}, \lambda_{S}$ and $\Phi$. Each solution corresponds to a state of equilibrium of the two-region system. Because the equations are nonlinear, a capacitor subject to a given total charge $Q$ may have multiple states of equilibrium. We regard the total charge as the control parameter, and plot these states of equilibrium in bifurcation diagrams: on the charge-voltage plane (Fig. 3d), the charge- $\lambda_{S}$ plane (Fig. 3e), and the charge- $\lambda_{L}$ plane (Fig. 3f). As the voltage is the same for both regions, (8) and (9) always have a trivial solution $\lambda_{S}=\lambda_{L}$, corresponding to a homogeneous state (black curves in Figs. 3d-3f). Two branches of inhomogeneous solutions exist, corresponding to $\lambda_{S}<\lambda_{L}$ (red curves) and $\lambda_{S}>\lambda_{L}$ (blue curves). To show the branches clearly, we use different scales for the ordinates in Figs. 3e and $3 \mathrm{f}$.

The multiplicity of solutions can be understood graphically. The voltage $\Phi$ is common for the two parallel capacitors, and the total charge is the sum $Q=Q_{L}+Q_{S}$. The charge-voltage relations for the two capacitors have the form $Q_{L}=A_{L} f\left(\Phi / H_{L}\right)$ and $Q_{S}=A_{S} f\left(\Phi / H_{S}\right)$, where the universal function $f$ is the same as that for the homogeneous system (Fig. 1c). For a given voltage below the maximum, each of the functions $Q_{L}(\Phi)$ and $Q_{S}(\Phi)$ gives two values of charge. The curves in Fig. 3d can be obtained by adding the charge-voltage curves of the two capacitors, $Q(\Phi)=Q_{L}(\Phi)+Q_{S}(\Phi)$. Thus, the resulting function $Q(\Phi)$ has four values of charge for each value of voltage. Furthermore, for the two capacitors of the same initial thickness, $H_{L}=H_{S}=H$, the maximum voltage for the two capacitors is the same as that for the homogeneous system, $\Phi_{c}$. The total charge at this voltage is the sum $Q_{L}+Q_{S}=\left(A_{L}+A_{S}\right) f\left(\Phi_{c} / H\right)=A f\left(\Phi_{c} / H\right)$, which is the same as the critical charge of the homogeneous system, $Q_{c}$. Thus, all four branches of solutions merge at the single-capacitor critical values $\left(Q_{c}, \Phi_{c}\right)$.

Imagine that we ramp up the total charge on the capacitor gradually. When the total 
charge is below the critical value, $Q<Q_{c}$, the two regions deform by the same stretch, along the curve of homogeneous deformation. When the total charge exceeds the critical value, $Q>Q_{c}$, the homogeneous deformation becomes unstable, and both branches of inhomogeneous states are stable. Consequently, the capacitor must switch from the homogeneous state to one of the inhomogeneous states. At the fixed charge $Q=Q_{c}$, the vertical purple lines represent the capacitor that snaps from the homogeneous state to a state with localized charge (blue curves). This localized state has a lower voltage (Fig. 3d), an expanded small region (Fig. 3e), and a contracted large region (Fig. 3 f). The switch from the homogeneous state to the localized state is a first-order transition, and greatly reduces the free energy of the capacitor. At $Q=Q_{c}$, the capacitor can also make another transition, without discontinuity in voltage or stretches of the two regions (red curves), of lower voltage, contracted small region, and expanded large region. This continuous transition reduces free energy only by a small amount and is therefore less favorable. We next focus on the snapping transition.

In the course of the snapping transition, charges flow from the large region to the small region, and this localization amplifies the electrical field in the small region, which may lead to electrical breakdown. We plot another bifurcation diagram in the plane of the fixed $Q$ and the electric field in the small region $E_{S}$, where we only include the stable region of the homogeneous branch (black) and the blue branch of the inhomogeneous state (Fig. 4a). Each blue curve corresponds to a given value of $A_{S} / A$, the vertical purple line corresponds to the snap, and the intersection between the blue curve and the purple line determines the electric field in the small region after the snap. The snap amplifies the electric field more if the relative size of the small region $A_{S} / A$ is smaller.

Assume that the breakdown electric field $E_{E B}$ is large enough so that the dielectric does not suffer electrical breakdown in the homogeneous state, $E_{E B}>E_{c}$, namely, 
$E_{E B}>2^{-2 / 3} \sqrt{3 \mu / \varepsilon}$. After the capacitor snaps into the inhomogeneous state, the electric field in the small region amplifies to $E_{S}$. This snap will cause electrical breakdown if $E_{S}>E_{E B}$. The electric field $E_{S}$ is a decreasing function of $A_{S} / A$. Consequently, whether the electromechanical localization will cause electrical breakdown depends on a combination of material properties, $E_{E B} \sqrt{\varepsilon / \mu}$, and the relative size of the snapping region, $A_{S} / A$. The two parameters form a plane, in which the condition $E_{S}=E_{E B}$ is a curve (Fig. 4b). Above this curve, the localization will not cause electrical breakdown. Below this curve, the localization will cause electrical breakdown.

We derive the asymptotic behavior of the localized state in the limit of small $A_{S} / A$. In this limit, the small region deforms greatly, $\lambda_{S}>>1$, while the large region is nearly undeformed, $\lambda_{L} \rightarrow 1$. Consequently, (8) and (9) give that $\lambda_{L} \approx 1+\lambda_{S}^{-2} / 6$ and $\Phi \approx H \sqrt{\mu / \varepsilon} \lambda_{S}^{-1}$. Substituting these two expressions into (6), replacing $Q$ by $Q_{c}=\sqrt{3 \varepsilon \mu} A$, and retaining the leading term, we obtain that $\lambda_{S} \approx 3^{1 / 6}\left(A_{S} / A\right)^{-1 / 3}$. The electric field in the small region is $E_{S}=\lambda_{S}^{2} \Phi / H \approx 3^{1 / 6} \sqrt{\mu / \varepsilon}\left(A_{S} / A\right)^{-1 / 3}$. This power law closely approximates the numerical solution for small $A_{S} / A$ (Fig. 4b). In this limit, the charge remaining in the large region is a small fraction of the total change on the capacitor, $Q_{L} / Q \approx 3^{-2 / 3}\left(A_{S} / A\right)^{1 / 3}$.

We are unaware of any direct experimental observation of charge localization. However, the instability of a homogeneous dielectric membrane has been predicted using a similar theoretical procedure, and has been verified by experimenats. ${ }^{31,34}$ The electromechanical localization is reminiscent of a well-known mechanical instability. When a metallic wire is pulled beyond a certain strain, homogenous deformation becomes unstable, and the wire forms a neck. This necking instability will set in even when the wire is in displacement-controlled tension. ${ }^{35}$ The neck will lead to fracture if the wire is long, but will stabilize if the wire is short. In the necking 
instability of long metal wires, the length of the neck is comparable to the diameter of the wire. Similarly, we expect that the electromechanical localization will occur over an area about $A_{S} \approx H^{2}$.

For a commonly used dielectric elastomer $\mathrm{VHB}^{\mathrm{TM}}$, the representative value is $E_{E B} \sqrt{\varepsilon / \mu}=4.73 .3^{1,36}$ The localized state does not undergo electrical breakdown if $A_{S} / A>0.016$. This condition translates to the area of the electrode below about $A \approx 61 H^{2}$, or the diameter of the electrode below about $D \sim 8 H$. For an initially $0.5 \mathrm{~mm}$ thick membrane, for example, the corresponding "breakdown-safe" linear size will be in the $\mathrm{mm}$ range. If the breakdown does not occur, the capacitor can be switched between the homogeneous and the localized state repeatedly. Upon this switch, the voltage drops significantly, and the small region deforms greatly. These characteristics can enable devices with bistable states, such as Braille displays. $^{8}$ Furthermore, the bistable states can be tuned and modified in many ways, such as by using a stiffening elastomer with a relatively small limiting stretch, applying a prestretch, introducing imperfections, and laminating the deformable capacitor (or part of it) with a passive soft layer.

In summary, when the electric charge is immobile on the surfaces of the dielectric, charge-controlled actuation is stable and does not suffer pull-in instability. In the presence of electrodes, however, charges will be mobile, and charge-controlled actuation is bistable. At a critical charge, the homogeneous deformation becomes unstable, and the capacitor will snap into a state of localized deformation by a first-order transition, which may lead to electrical breakdown. However, the breakdown in the charge-localization region can be avoided if the initial area of the electrodes is small. This bistability is tunable and can be used to design devices.

\section{Acknowledgments}

The work at Harvard was supported by ARO (W911NF-09-1-0476), DARPA 
(W911NF-10-1-0113), and MRSEC (DMR-0820484). T.L. was supported by China Scholarship Council as a visiting scholar for two years at Harvard University. Z.S. acknowledges a visiting appointment at the International Center for Applied Mechanics. The work in Linz was supported by the FWF project I-986-N20 and by the European Research Council (ERC Advanced Investigators Grant ‘Soft-Map’).

\section{References}

1 F. Iida, and C. Laschi, Procedia Comput. Sci. 7, 99 (2011).

2 G. Kofod, W. Wirges, M. Paajanen, and S. Bauer, Appl. Phys. Lett. 9o, o81916 (2007).

3 S. A. Morin, R. F. Shepherd, S. W. Kwok, A. A. Stokes, A. Nemiroski, and G. M. Whitesides, Science 337, 828 (2012).

4 S. Wagner, and S. Bauer, MRS Bulletin 37, 207 (2012).

5 S. Kim, C. Laschi, and B. Trimmer, Trends Biotechnol. 31, 287 (2013).

6 R. Pelrine, R. Kornbluh, Q. B. Pei, and J. Joseph, Science 287, 836 (2000).

7 F. Carpi, S. Bauer, and D. D. Rossi, Science 330, 1759 (2010).

8 P. Brochu, and Q. B. Pei, Macromol. Rapid. Commun. 31, 10 (2010).

9 Q. B. Pei, R. Pelrine, S. Stanford, R. Kornbluh, and M. Rosenthal, Synthetic Metals 135-136, 129 (2003).

10 I.A. Anderson, T.A. Gisby, T. McKay, B.M. O’Brien, and E. Calius, J. Appl. Phys. 112, 041101 (2012).

11 R. Shankar, T. K. Ghosh, and R. J. Spontak, Adv. Mater. 19, 2218 (2007).

12 G. Kovacs, L. Düring, S. Michel, and G. Terrasi, Sens. Actuators A 155, 299 (2009).

13 S. Rosset, M. Niklaus, P. Dubois, and H.R. Shea, J. Microelectromechanical Sys. 18, 1300 (2009).

14 F. Carpi, G. Frediani, S. Turco, and D. D. Rossi, Adv. Func. Mater. 21, 4002 (2011).

15 S. Shian, R. M. Diebold, and D. R. Clarke, Opt. Express 21, 8669 (2013).

16 A. Cheng, WW-EAP Newsletter 13, 2 (2011). 
17 C. Keplinger, J.Y. Sun, C.C. Foo, P. Rothemund, G.M. Whitesides, Z. Suo, Science 341, 984(2013).

18 T. Mckay, B. O'Brien, E. Calius, and I. Anderson, Appl. Phys. Lett. 97, 062911 (2010).

19 R. Kaltseis, C. Keplinger, R. Baumgartner, M. Kaltenbrunner, T.F. Li, P. Machler, R. Schwodiauer, Z. G. Suo, and S. Bauer, Appl. Phys. Lett.99, 162904 (2011).

20 R. D. Kornbluh, R. Pelrine, H. Prahlad, A. Wong-Foy, B. McCoy, S. Kim, J. Eckerle, and T. Low, MRS Bulletin 37, 246 (2012).

21 J. S. Huang, S. Shian, Z. G. Suo, and D. R. Clarke, Adv. Funct. Mater. 2013, DOI: $10.1002 / \mathrm{adfm} .201300402$.

22 X. H. Zhao and Z. G. Suo, Appl. Phys. Lett. 91, 061921 (2007).

23 X. H. Zhao, and Z. G. Suo, Phys. Rev. Lett. 104, 178302 (2010).

24 C. Keplinger, M. Kaltenbrunner, N. Arnold, and S. Bauer, PNAS 107, 4505-4510 (2010).

25 B. Li, J. X. Zhou, and H. L. Chen, Appl. Phys. Lett. 99, 244101 (2011).

26 Z.G. Suo, Acta Mechanica Solida Sinica 23, 549 (2010).

27 X. H. Zhao, W. Hong, and Z. G. Suo, Phys. Rev. B 76, 134113 (2007).

28 S. J. A. Koh, T. F. Li, J. X. Zhou, X. H. Zhao, W. Hong, J. Zhu, and Z. G. Suo, J.Polym. Sci. Part B: Polym. Phys. 49, 504 (2011).

29 C. Keplinger, T. F. Li, R. Baumgartner, Z. Suo, and S. Bauer, Soft Matter 8, 285 (2012).

30 T. Q. Lu and Z. G. Suo, Acta Mechanica Sinica 28, 1106 (2012).

31 T. Q. Lu, J. S. Huang, C. Jordi, K. Gabor, R. Huang, D. R. Clarke, and Z. G. Suo, Soft Matter 8, 6167 (2012).

32 R. Huang, and Z. G. Suo, Proc. Roy. Soc. A 468, 1014 (2012).

33 M. Kollosche, J. Zhu, Z. G. Suo, and G. Kofod, Phys. Rev. E 85, 051801 (2012).

34 T. F. Li, C. Keplinger, R. Baumgartner, S. Bauer, W. Yang, and Z. G. Suo, J. Mech. Phys. Solids 61, 611 (2013).

35 J. W. Hutchinson, and K. W. Neale, Acta Metal. 25, 839 (1977).

36 G. Kofod, R. Kornbluh, R. Pelrine, and P. S. Larsen, J. Intel. Mater. Sys. Struct. 14, 787 (2003). 


\section{Figure Captions}

FIG. 1. Homogeneous deformation. (a) Free energy for charge-controlled actuation. (b) Free energy for voltage-controlled actuation. (c) States of equilibrium plotted on the charge-voltage plane.

FIG. 2. Localized deformation. (a) When the membrane with compliant electrodes is charged beyond a critical value, the homogeneous deformation is unstable, and a small region of the membrane deforms more than the rest of the membrane. (b) A model of localization represents the membrane by two regions subject to the same voltage, but each region undergoes a separate homogeneous deformation.

FIG. 3. First-order transition in a charge-controlled capacitor. The relative size of the small region is $A_{S} / A=0.1$. The contours of the free energy function $F\left(\lambda_{L}, \lambda_{S}\right)$ for three values of the total charge: (a) $Q /(A \sqrt{\varepsilon \mu})=1$, (b) $Q /(A \sqrt{\varepsilon \mu})=1.3$, (c) $Q /(A \sqrt{\varepsilon \mu})=2.5$. Bifurcation diagrams show branches of states of equilibrium. The horizontal axis is the control parameter, the total charge on the capacitor. The vertical axis is (d) the voltage, (e) the stretch of the small region $\lambda_{S}$, and (f) the stretch of the large region $\lambda_{L}$. The black curve represents the homogeneous deformation, while the blue and red curves represent two branches of inhomogeneous deformation. The vertical purple arrow marks the snapping transition from the homogeneous state to the localized state.

FIG. 4. Electromechancial localization and electrical breakdown. (a) Bifurcation diagram plotted on the plane of the total charge $Q$ and the electric field in the small part $E_{S}$. (b) The condition under which electromechanical localization does not cause electrical breakdown. 


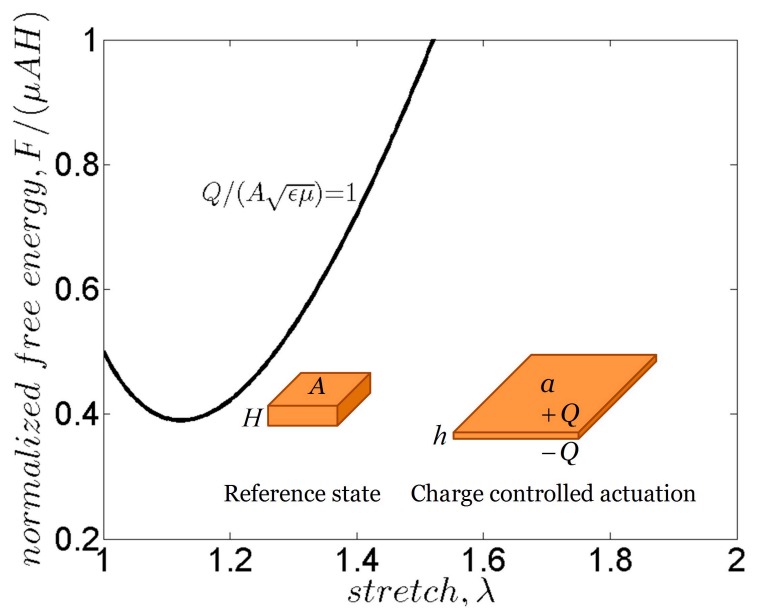

(a)

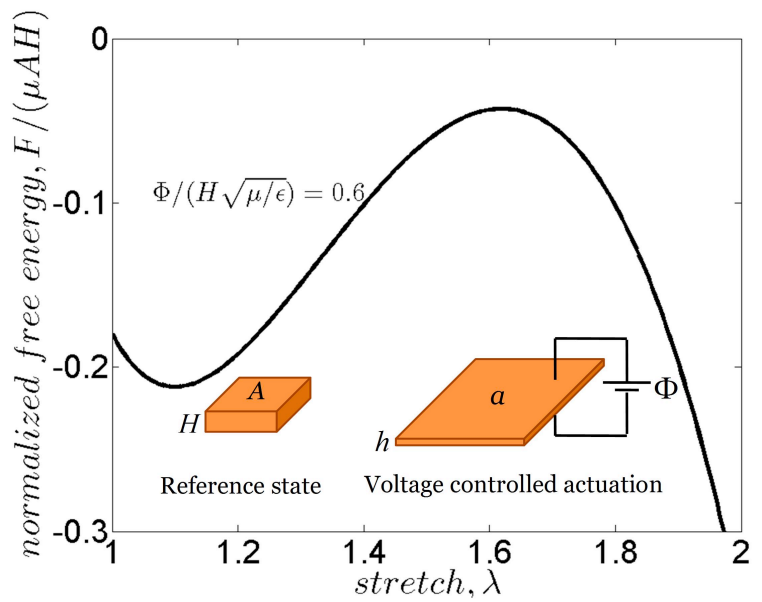

(b)

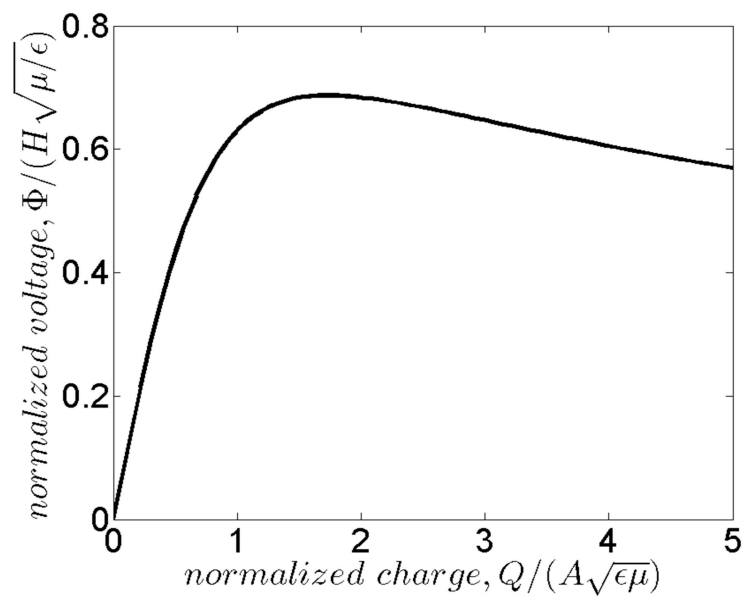

(c)

FIG. 1. Homogeneous deformation. (a) Free energy for charge-controlled actuation. (b) Free energy for voltage-controlled actuation. (c) States of equilibrium plotted on the charge-voltage plane. 


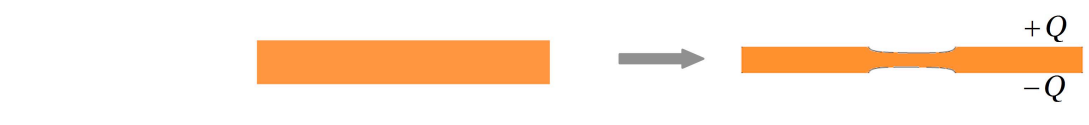

(a)

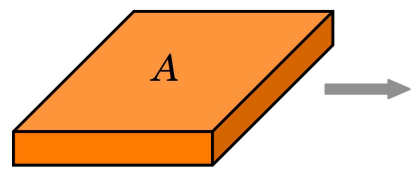

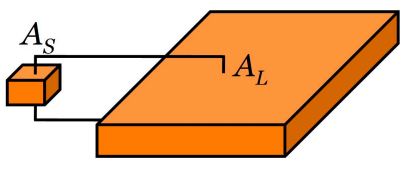

Reference state

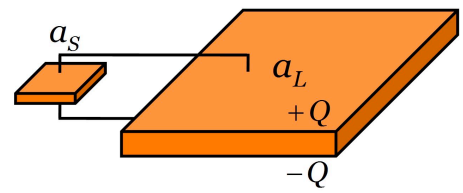

Actuated state

(b)

FIG. 2. Localized deformation. (a) When the membrane with compliant electrodes is charged beyond a critical value, the homogeneous deformation is unstable, and a small region of the membrane deforms more than the rest of the membrane. (b) A model of localization represents the membrane by two regions subject to the same voltage, but each region undergoes a separate homogeneous deformation. 


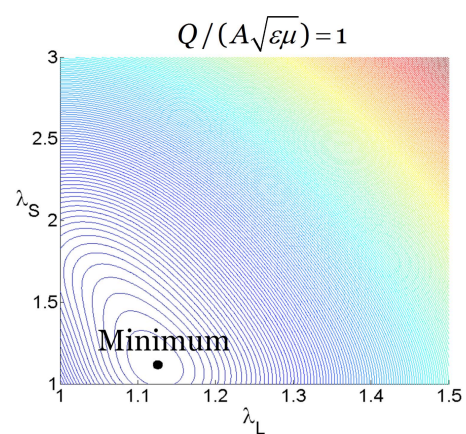

(a)

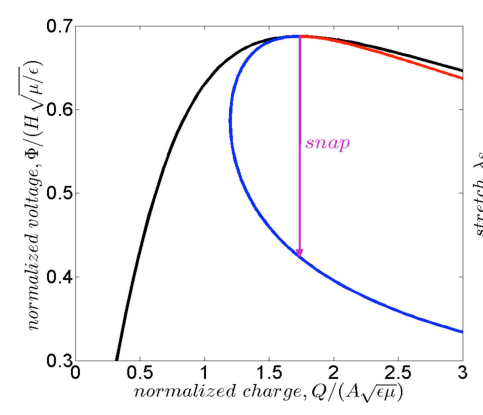

(d)

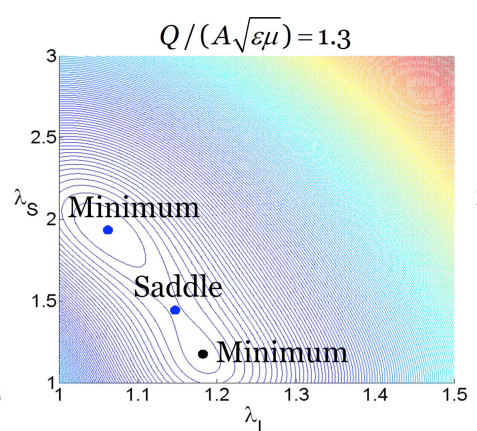

(b)

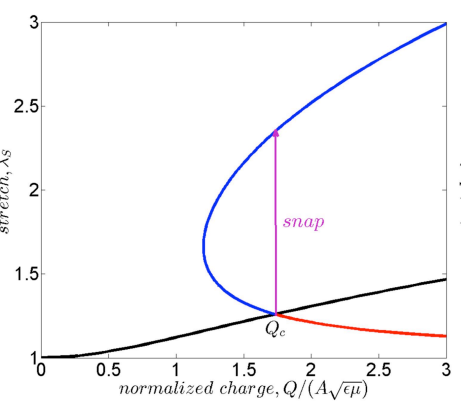

(e)

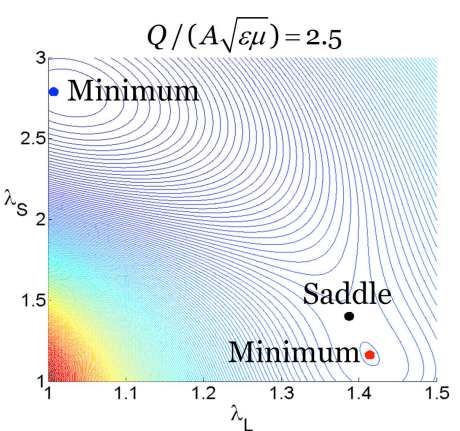

(c)

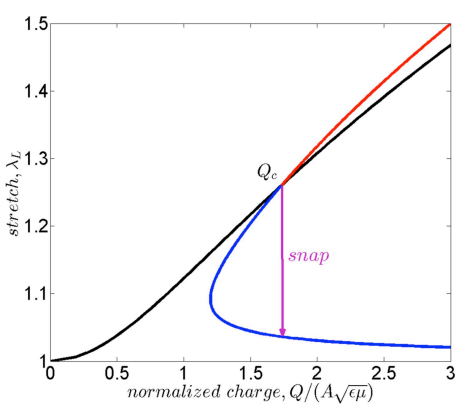

(f)

FIG. 3. First-order transition in a charge-controlled capacitor. The relative size of the small region is $A_{S} / A=0.1$. The contours of the free energy function $F\left(\lambda_{L}, \lambda_{S}\right)$ for three values of the total charge: (a) $Q /(A \sqrt{\varepsilon \mu})=1$, (b) $Q /(A \sqrt{\varepsilon \mu})=1.3$, (c) $Q /(A \sqrt{\varepsilon \mu})=2.5$. Bifurcation diagrams show branches of states of equilibrium. The horizontal axis is the control parameter, the total charge on the capacitor. The vertical axis is (d) the voltage, (e) the stretch of the small region $\lambda_{S}$, and (f) the stretch of the large region $\lambda_{L}$. The black curve represents the homogeneous deformation, while the blue and red curves represent two branches of inhomogeneous deformation. The vertical purple arrow marks the snapping transition from the homogeneous state to the localized state. 


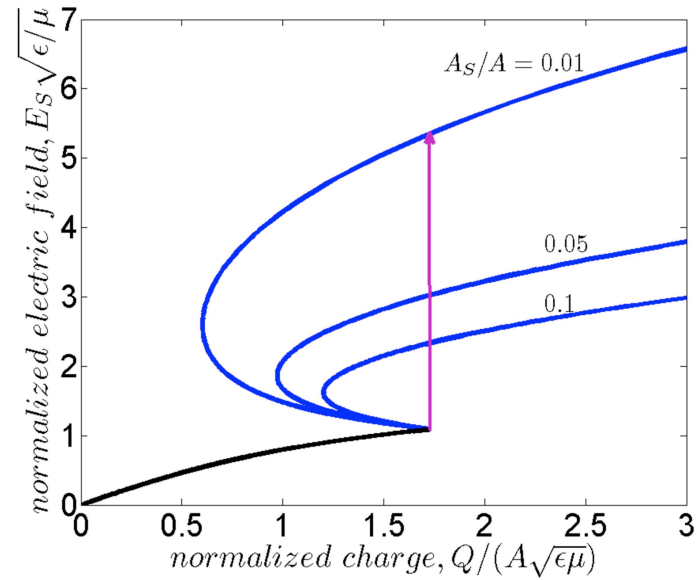

(a)

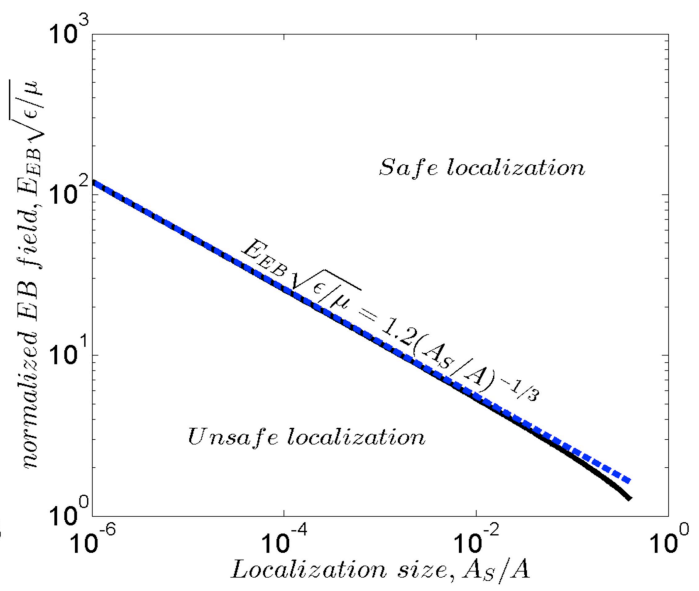

(b)

FIG. 4. Electromechancial localization and electrical breakdown. (a) Bifurcation diagram plotted on the plane of the total charge $Q$ and the electric field in the small part $E_{S}$. (b) The condition under which electromechanical localization does not cause electrical breakdown. 